\title{
Endocarditis infecciosa izquierda por Pseudomonas aeruginosa tratada médicamente
}

\author{
Karen Melissa Ordóñez, Odismar Andrea Hernández, Jorge Alberto Cortés, María José López, \\ Gladys Alfonso, Alejandro Junca \\ Departamento de Medicina Interna, Facultad de Medicina, Universidad Nacional de Colombia, \\ Bogotá, D.C., Colombia
}

\begin{abstract}
La endocarditis infecciosa por Pseudomonas aeruginosa es una entidad poco frecuente, de difícil diagnóstico y alta mortalidad. Se presenta a continuación el caso de un hombre de 51 años, sin antecedentes de uso de drogas intravenosas ni enfermedad valvular, con antecedentes de colecistectomía en el mes anterior, quien se presentó a urgencias con un cuadro febril asociado a síntomas gastrointestinales, y, posteriormente mostró signos de embolismo a distancia, hemocultivos positivos para $P$. aeruginosa y desarrollo de múltiples complicaciones propias de la enfermedad.

La presentación clínica de la endocarditis infecciosa es inespecífica, lo cual genera diagnósticos tardíos que impiden la instauración de un tratamiento precoz y eficaz, como el reemplazo valvular, indicado en endocarditis por hongos o por gérmenes como $P$. aeruginosa. Este caso es fortuito por su resolución solamente con tratamiento médico combinado, con amikacina y meropenem, ya que tuvo varias complicaciones que contraindicaron el manejo quirúrgico.
\end{abstract}

Palabras clave: endocarditis, P. aeruginosa, válvula aórtica, embolia, terapia combinada, mortalidad.

Left-sided infective endocarditis caused by Pseudomonas aeruginosa treated medically

Infective endocarditis due to Pseudomonas aeruginosa is a rare clinical condition, difficult to diagnose and associated with high mortality. Herein we present a case of a 51 years old male without history of intravenous drug use or valvular disease, with past medical history of cholecystectomy in the previous month, who presented to the emergency department with fever, gastrointestinal symptoms, and subsequent signs of distant embolization, positive blood cultures for $P$. aeruginosa and development of multiple complications of the disease. The clinical presentation of infective endocarditis is nonspecific, leading to delayed diagnosis, and preventing early and effective treatment. Valvular replacement is indicated in fungal or $P$. aeruginosa endocarditis. This case is notable because of the resolution with amikacin combined with meropenem, in a patient with several complications that contraindicated surgery.

Key words: endocarditis, Pseudomonas aeruginosa, aortic valve, embolism, combined modality therapy, mortality.

La endocarditis infecciosa por Pseudomonas aeruginosa es una entidad que se ha descrito, principalmente en usuarios de drogas intravenosas, con compromiso de las válvulas tricúspide y pulmonar $(1,2)$. Este tipo de endocarditis presenta una buena respuesta al tratamiento médico, a diferencia de la endocarditis de cavidades izquierdas con compromiso de las válvulas aórtica y mitral, cuyo tratamiento de elección es el reemplazo valvular dada la alta tasa de mortalidad descrita con el tratamiento médico (3-5).

\section{Correspondencia:}

Karen Melissa Ordóñez, Calle 74 № 108-50, Bogotá, D.C., Colombia.

Teléfono: (311) 4894658

karenmelissao@gmail.com

Recibido: 09/06/09; aceptado:19/11/09
A continuación presentamos un caso de endocarditis infecciosa hospitalaria por $P$. aeruginosa, con compromiso de la válvula aórtica, complicada con émbolos sépticos al sistema nervioso central y adecuada respuesta a la terapia antibiótica, seguida de una revisión sobre endocarditis infecciosa.

\section{Reporte de caso}

Se trata de un paciente de sexo masculino, de 51 años que ingresó a urgencias por 10 días de evolución de fiebre de $40^{\circ} \mathrm{C}$, asociada a diaforesis y deposiciones líquidas sin moco ni sangre.

Tenía antecedentes de colecistectomía por laparoscopia 41 días antes, que requirió hospitalización por 31 días para el tratamiento de una infección del sitio operatorio con compromiso de órgano/espacio, tratada con meropenem. No 
se había realizado monitorización hemodinámica invasiva y no tenía antecedentes de uso de drogas ilícitas intravenosas.

En el examen físico se encontraba alerta, orientado, con frecuencia cardiaca de 72 latidos por minuto, frecuencia respiratoria de 16 respiraciones por minuto, tensión arterial de $95 / 57 \mathrm{~mm} \mathrm{Hg}$, temperatura de $38,5^{\circ} \mathrm{C}$ y deshidratación grado I. Se auscultó un soplo holosistólico en el foco aórtico, grado II/VI. En el abdomen había dolor a la palpación en epigastrio, sin signos de irritación peritoneal.

En los exámenes de laboratorio se encontró preservación de la función renal, anemia normocítica, normocrómica, sin leucocitosis ni neutrofilia en el hemograma, elevación de la proteína $C$ reactiva (PCR) y leve aumento de las transaminasas, y fosfatasa alcalina y amilasa en rango normal. Además, se realizó una ecografía abdominal que mostró distensión de asas intestinales y esplenomegalia. Los antígenos de superficie de la hepatitis $B$ y los anticuerpos para la hepatitis $C$ fueron negativos.

En el sexto día de hospitalización, el paciente persistía febril, con elevación de reactantes de fase aguda y signos de respuesta inflamatoria sistémica; preliminarmente, se reportaron dos hemocultivos que mostraron crecimiento de bacilos Gram negativos. En este momento, se inició manejo antibiótico con 4,5 g intravenosos de piperacilina/tazobactam cada seis horas, bajo la impresión diagnóstica de colangitis.

Después del inicio de la terapia antibiótica, se observó mejoría en las pruebas de función hepática, pero aumentó el valor de la PCR y hubo persistencia de los signos de respuesta inflamatoria sistémica (taquicardia y fiebre) con trombocitopenia. Para hacer la monitorización hemodinámica, se decidió colocar un catéter venoso central.

El noveno día de hospitalización, en el examen físico se encontraron pápulas hiperpigmentadas en palmas y plantas, hemorragia subconjuntival derecha y un nódulo subcutáneo doloroso en el codo izquierdo (figura 1).

El décimo día de hospitalización, los hemocultivos mostraron crecimiento de $P$. aeruginosa con sensibilidad intermedia a imipenem [concentación inhibitoria máimima (CIM) de 8], sensible a amikacina $(C I M<4)$ y piperacilina/tazobactam $(C I M<8)$. Seajustó la terapia antibiótica a meropenem más amikacina. Se practicó una tomografía computadorizada

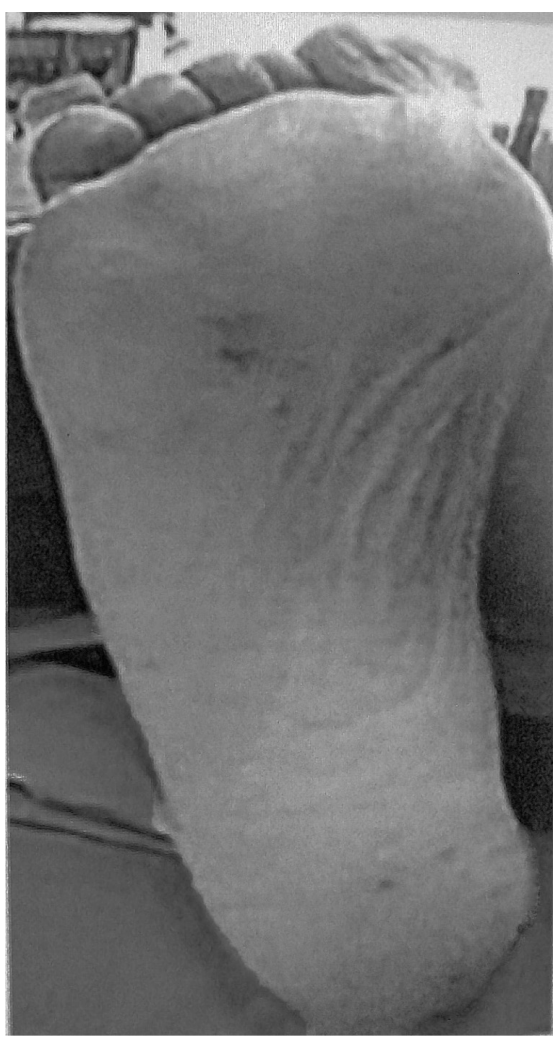

Figura 1. Signos clínicos de endocarditis, pápulas hiperpigmentadas dolorosas en planta de pie.

abdominal que mostró esplenomegalia e imágenes hipodensas circunscritas en su interior.

En el decimosegundo día de hospitalización, presentó paresia del miembro inferior izquierdo, por lo cual se practicó una tomografía computadorizada cerebral que mostró lesiones hipodensas occipitales y parietales bilaterales. En este momento, un ecocardiograma transesofágico demostró una vegetación móvil de $5 \mathrm{~mm}$ en la válvula aórtica.

El paciente continuó con signos de respuesta inflamatoria sistémica y elevación progresiva de las transaminasas y, para el decimocuarto día de hospitalización, presentó deterioro súbito de la conciencia, hemiparesia izquierda, palidez, diaforesis y signo de Babinski izquierdo; en la tomografía computadorizada cerebral se observaron múltiples focos hemorrágicos corticales y córtico-basales, con drenaje al ventrículo lateral derecho. Se practicó intubación orotraqueal para proteger la vía aérea.

En el decimosexto día de hospitalización, presentó deterioro clínico dado por oliguria, extracción de oxígeno aumentada y descenso significativo en los niveles de hemoglobina. Se inició soporte inotrópico 
con dobutamina. No presentó fiebre posteriormente y, al día siguiente, presentó mejoría neurológica sin sedación, hemiplejía izquierda y afasia motora con una escala de Glasgow que mejoró hasta 13/15, por lo cual se retró el tubo y no requirió soporte inotrópico.

Se recibieron dos hemocultivos posteriores al inicio del tratamiento con meropenem y amikacina, que informaron crecimiento de $P$. aeruginosa en el primero y ningún crecimiento de colonias en el segundo.

Al día 30 de hospitalización, presentó resolución de la afasia, sin recuperación completa de la fuerza del hemicuerpo izquierdo y sin nuevos signos de respuesta inflamatoria sistémica. Se completaron 42 días de tratamiento antibiótico combinado.

\section{Discusión}

La endocarditis infecciosa es una enfermedad poco frecuente, con una incidencia de 1,5 a 6 casos por 100.000 personas-año. Los principales factores de riesgo asociados a su presentación son: cardiopatía congénita cianosante, comunicación interventricular, conducto arterioso permeable o presencia de válvulas protésicas, tratamiento de hemodiálisis, dispositivos invasivos y uso de drogas intravenosas (6).

Las primeras publicaciones sobre endocarditis infecciosa datan de 1885, cuando se describió como una entidad de difícil diagnóstico y con una letalidad cercana al 100\% (7). En los últimos 30 años y con el fin de realizar un diagnóstico más temprano, se han propuesto criterios como los de Reyn et al., en 1982, y los de Durack et al., en 1994, con una sensibilidad para el diagnóstico de $51 \%$ y $80 \%$, respectivamente $(8,9)$.

La presentación clínica de la endocarditis es muy variada, lo cual dificulta su diagnóstico oportuno. En los casos descritos, los síntomas más frecuentes son: escalofríos, astenia y disnea $(40 \%$ cada uno), diaforesis, anorexia, pérdida de peso y tos ( $25 \%$ cada uno), focalización neurológica, náuseas y cefalea ( $20 \%$ cada uno), mialgias, dolor abdominal, artralgias y dolor torácico (15\% cada uno), y hemoptisis (10\%). Entre los signos, el más frecuente es la fiebre $(90 \%)$, seguida de soplo cardiaco (85\%), embolismo (50\%), esplenomegalia (57\%), aneurismas micóticos $(20 \%)$ y lesiones en retina $(10 \%)(10)$.

Dado lo inespecífico de su presentación, la sospecha clínica con base en los factores de riesgo y la presencia de fiebre, son esenciales para el diagnóstico temprano de esta entidad, así como para la prevención de complicaciones asociadas. En nuestro paciente, las principales manifestaciones clínicas, además de la fiebre, fueron los síntomas gastrointestinales que, en el contexto de un periodo postoperatorio, desviaron el enfoque clínico hacia una alteración abdominal; la endocarditis infecciosa se consideró sólo cuando las manifestaciones de embolismo eran ya evidentes.

El ecocardiograma es el pilar fundamental para el diagnóstico de la endocarditis infecciosa. Se recomienda la realización del ecocardiograma transesofágico por su mejor rendimiento para el diagnóstico respecto al ecocardiograma transtorácico: sensibilidad de $48 \%$ a $100 \%$ para el primero, y de $18 \%$ a $63 \%$ para el segundo, así como por una mejor resolución en la identificación de estructuras como abscesos, dehiscencias, trombos en la aurícula izquierda, vegetaciones menores de $5 \mathrm{~mm}$ y lesiones en la pared posterior de las válvulas protésicas (11-13).

Otro elemento fundamental para el diagnóstico de la endocarditis infecciosa es el hemocultivo. En las series descritas, se ha documentado el aislamiento del agente etiológico en $95 \%$ de los casos. Para optimizar el desempeño de la prueba, en la endocarditis se recomienda realizar dos tomas de $10 \mathrm{ml}$ en sitios diferentes de venopunción (14).

Los agentes causales de la endocarditis infecciosa varían según el tipo de población y los factores de riesgo. En los pacientes con endocarditis infecciosa adquirida en la comunidad, los agentes más frecuentemente aislados son: Streptococcus spp., Staphylococcus aureus y Enterococcus spp. En los usuarios de drogas intravenosas, los principales agentes descritos son: $S$. aureus, Staphylococcus coagulasa negativa, estreptococos del grupo viridans, Enterococcus spp., P. aeruginosa y Candida spp.

En los pacientes con endocarditis infecciosa hospitalaria, los agentes principales son $S$. aureus, Staphylococcuscoagulasa negativa y Enterococcus spp. (15-17). El aislamiento de $P$. aeruginosa en nuestro paciente puede explicarse por la estadía prolongada en el medio hospitalario y la exposición a antibióticos, factores asociados a la colonización e infección por esta bacteria (18).

El tratamiento y el pronóstico de la endocarditis infecciosa por $P$. aeruginosa dependen de las cavidades afectadas y de la presencia de 
complicaciones, como falla cardiaca, abscesos perianulares, émbolos sépticos a distancia y aneurismas por hongos.

En la endocarditis de cavidades derechas, la densidad de las bacterias en las vegetaciones es menor que en la de las cavidades izquierdas, lo cual aumenta las probabilidades de éxito terapéutico con el tratamiento médico.

Con el compromiso de las cavidades izquierdas, en cambio, el reemplazo valvular es el tratamiento de elección (19). Bicanic (20) describió una búsqueda en la literatura de 17 casos de endocarditis infecciosa de cavidades izquierdas por $P$. aeruginosa en válvulas nativas, de los cuales sólo cinco sobrevivieron $(29,4 \%)$, todos con tratamiento quirúrgico. P. Gavin (21), en una revisión de casos de endocarditis infecciosa izquierda por $P$. aeruginosa, halló 9 de 26 casos (34\%) con éxito terapéutico. Los esquemas antibióticos utilizados en los casos reportados incluyeron penicilinas, cefalosporinas, carbapenem y aminoglucósidos, incluso medicamentos sin actividad contra Pseudomonas spp. in vitro $(20,21)$.

Entre las recomendaciones actuales para el tratamiento médico, se encuentra la combinación de un betalactámico con un aminoglucósido. Para evaluar la efectividad clínica de esta combinación, se han realizado varios estudios, tanto en pacientes inmunocompetentes como inmunocomprometidos.

Paul et al. (22) realizaron una revisión de 64 estudios con 7.586 pacientes sépticos inmunocompetentes, para comparar la mortalidad, los efectos secundarios y el desarrollo de resistencia bacteriana entre los pacientes tratados con monoterapia con betalactámicos, con respecto a los tratados con la combinación con aminoglucósidos. En el análisis no se encontraron diferencias en la mortalidad o en el fracaso clínico, pero se observó una mayor nefrotoxicidad con la combinación. En el análisis de los subgrupos para infecciones por $P$. aeruginosa y cocos Gram positivos, tampoco se encontraron diferencias significativas.

Falagas et al. (23) hicieron una revisión de cinco estudios con 261 pacientes, para evaluar la efectividad de la combinación específicamente en endocarditis infecciosa, y obtuvieron resultados similares al metanálisis de Paul. El único metanálisis que favoreció la terapia combinada fue el de Safdar et al. (24), en el cual se revisaron 17 estudios para evaluar si este esquema reducía la mortalidad en bacteriemia por Gram negativos y se encontró un beneficio para pacientes infectados por $P$. aeruginosa.

Las conclusiones de los metanálisis descritos aportan una valiosa información sobre la terapia combinada en infecciones graves en pacientes inmunocompetentes. Sin embargo, debe aclararse que los estudios incluidos para el análisis son heterogéneos, escasos y antiguos, algunos con antibióticos y dosis que actualmente no se usan, lo cual debe tenerse en cuenta en su análisis. Por otro lado, los hallazgos en $P$. aeruginosa no son concluyentes, ya que fueron muy pocos los estudios incluidos. Al ser la terapia combinada la recomendación vigente y la utilizada en los casos reportados en la literatura, iniciamos en nuestro paciente la combinación de meropenem con amikacina.

A pesar de la sensibilidad de $P$. aeruginosa a piperacilina/tazobactam, se decidió cambiar el tratamiento a meropenem por la persistencia de signos de respuesta inflamatoria sistémica a las 72 horas y por la discordancia encontrada entre la sensibilidad in vitro a este antibiótico y su actividad bactericida (25). La selección de meropenem en lugar del imipenem se hizo con base en el antibiograma, que mostró sensibilidad intermedia a éste, así como por la menor asociación con desarrollo de episodios convulsivos en pacientes con lesiones en el sistema nervioso central. Se decidió suministrarlo en infusión continua, para disminuir la probabilidad de desarrollo de resistencia durante el tratamiento (26).

Una alternativa para el tratamiento de infecciones graves por $P$. aeruginosa es doripenem. Este carbapenem tiene como ventaja sobre los demás una menor inducción de resistencia in vitro, además de contar con un bajo poder inductor de convulsiones y estabilidad en soluciones, como meropenem, que permite su administración en infusión de cuatro horas. Por el momento, este antibiótico se encuentra aprobado para el tratamiento de neumonía hospitalaria, infecciones intraabdominales e infecciones graves de las vías urinarias. Sería interesante conocer su comportamiento en infecciones del sistema nervioso central, para el tratamiento de la meningitis hospitalaria $(27,28)$.

Los casos de endocarditis infecciosa por $P$. aeruginosa favorecen el tratamiento quirúrgico sobre el tratamiento médico, aunque persista una mortalidad significativa. La mortalidad asociada al procedimiento quirúrgico en la endocarditis se 
encuentra entre $8 \%$ y $16 \%$, con una supervivencia a cinco años de $76 \%$ y a 10 años de $61 \%$ (29). La indicación y el momento de realización de la cirugía dependen de la presencia de complicaciones asociadas. La principal indicación es el desarrollo de falla cardíaca moderada a grave (New York Heart Association III-IV) secundaria a insuficiencia valvular. La cirugía debe practicarse de urgencia tan pronto se instaure el cuadro, dado que la mortalidad aumenta de $11 \%$ a $33 \%$ con el empeoramiento de la clase funcional $(30,31)$.

Entre las recomendaciones actuales se encuentra practicar un reemplazo valvular a aquellos pacientes que hayan presentado dos episodios mayores de embolismo durante el tratamiento o a aquéllos en quienes se encuentre una vegetación móvil o en la valva anterior de la válvula mitral.

En caso de complicaciones del sistema nervioso central, como ataque isquémico transitorio, ataque cerebrovascular embólico con hemorragia o sin ella, aneurismas micóticos rotos, meningitis y encefalopatía sin focalización, existe controversia sobre el momento indicado para la realización del procedimiento quirúrgico. En la actualidad, en caso de ataque cerebrovascular isquémico secundario a un émbolo séptico, se recomienda practicar la cirugía en las primeras 72 horas de identificada la lesión, así como postergarla por tres semanas en caso de demostrarse lesiones hemorrágicas $(32,33)$.

Los pacientes con endocarditis infecciosa pueden hacer embolismos sépticos a cualquier órgano. Su presencia debe sospecharse ante la persistencia de la fiebre a los siete días de tratamiento médico o la presencia de hemocultivos positivos. Uno de los órganos comprometidos con mayor frecuencia es el bazo. En caso de comprobarse el compromiso de este órgano, se sugiere practicar esplenectomía para favorecer la resolución del proceso infeccioso y disminuir la mortalidad asociada (34).

Este paciente se reporta por tratarse de un caso fortuito, en el cual una entidad con importante mortalidad y difícil diagnóstico presentó, como lo describe la literatura, múltiples complicaciones que contraindicaron el reemplazo valvular temprano. Probablemente, el efecto del embolismo paradójicamente permitió que el antibiótico penetrara más fácilmente a la vegetación y tuviese un desenlace favorable sólo con el tratamiento médico.

En conclusión, debe considerarse el diagnóstico de endocarditis infecciosa cuando no se identifique un foco infeccioso claro o ante una evolución clínica tórpida, con el fin de brindar el mejor tratamiento posible antes de que se desarrollen complicaciones que lo contraindiquen.

\section{Conflicto de intereses}

Los autores declaran no presentar conflicto de interés.

\section{Financiación}

Este artículo fue realizado en el tiempo laboral de Jorge Alberto Cortés y Gladys Alfonso, profesores de la Universidad Nacional de Colombia.

\section{Referencias}

1. Reisberg B. Infective endocarditis in the narcotic addict. Prog Cardiovasc Dis. 1979;22:193-204.

2. Wieland $M$, Lederman MM, Kline-King C, Kevs TF, Lerner PI, Bass SN, et al. Left-sided endocarditis due to Pseudomonas aeruginosa. A report of 10 cases and review of the literature. Medicine (Baltimore). 1986;65:180-9.

3. Reyes MP, Palutke WA, Wylin RF, Lerner AM. Pseudomonas endocarditis in the Detroit Medical Centre 1969-72. Medicine. 1973;52:173-94.

4. Witchitz S, Gilbert C, Witchitz J, Gougerot M, Vachon F, Vic-Dupont V. Pseudomonas aeruginosa endocarditis. A report of nine cases. Eur J Cardiol. 1976;4:91-7.

5. Finkelstein R, Boulus M, Markievicz M. Hospital-acquired Pseudomonas aeruginosa endocarditis. J Hosp Infect. 1991;18:161-3.

6. Berlin JA, Abrutyn E, Strom BL, Kinman JL, Levison ME, Korseniowski OM, et al. Incidence of infective endocarditis in the Delaware Valley 1988-1990. Am J Cardiol. 1995; 76:933-6.

7. Durack DT. Infective endocarditis. Infect Dis Clin N Am. 2002;16:xv-xix.

8. von Reyn CF, Levy BS, Arbeit RD, Friedland G, Crumpacker CS. Infective endocarditis: an analysis based on strict case definitions. Ann Intern Med. 1981; 94:505-18.

9. Bayer AS, Ward JI, Ginzton LE, Shapiro SM. Evaluation of new clinical criteria for the diagnosis of infective endocarditis. Am J Med. 1994;96:211-9.

10. Pelletier LL, Petersdorf RG. Infective endocarditis: a review of 125 cases from the University of Washington Hospitals, 1963-72. Medicine. 1977;56:287-313.

11. Sachdev M, Peterson G, Jollis J. Imaging techniques for diagnosis of infective endocarditis. Infect Dis Clin N Am. 2002:16:319-37.

12. Chirillo F, Bruni A, Giujusa T, Totis O, Cavarzerani A, Stritoni P. Echocardiography in infective endocarditis: reassesment of the diagnostic criteria of vegetation as evaluated from the precordial and transesophageal approach. Am J Card Imaging. 1995;9:174-9.

13. Morguet AJ, Werner GS, Andreas S, Kreuzer $\mathbf{H}$. Diagnostic value of transesophageal compared with 
transthoracic echocardiography in suspected prosthetic valve endocarditis. Herz. 1995;20:390-8.

14. Benn M, Hagelskjaer LH, Tvede M. Infective endocarditis, 1984 through 1993: a clinical and microbiological survey. J Intern Med. 1997;242:15-22.

15. Miró J, del Río A, Mestres C. Infective endocarditis in intravenous drug abusers and HIV-1 infected patients. Infect Dis Clin N Am. 2002;16:273-95.

16. Fernández N, Almirante B, Tornos P, Pigrau C, Sambola A, Igual A, et al. Contemporary epidemiology and prognosis of health-care associated infective endocarditis. Clin Infect Dis. 2008;47:1287-97.

17. Giannitsioti E, Skiadas I, Antoniadou A, Tsiodras $\mathrm{K}$, Kanavos $\mathrm{H}$, Triantafyllidi $\mathbf{H}$, et al. Nosocomial vs. community-acquired infective endocarditis in Greece: changing epidemiological profile and mortality risk. Clin Microbiol Infect. 2007;13:736-69.

18. Schechner V, Nobre V, Kaye K, Leshno M, Giladi M, Rohner $\mathbf{P}$, et al. Gram-negative bacteremia upon hospital admission: when should Pseudomonas aeruginosa be suspected? Clin Infect Dis. 2009;48:580-6.

19. Bayer AS, Norman DC. Valve-site specific pathogenic differences between right-sided and left-sided bacterial endocarditis. Chest. 1990;98:200-5

20. Bicanic TA, Eykyn SJ. Hospital-acquired, native valve endocarditis caused by Pseudomonas aeruginosa. J Infect. 2002;44:137-9

21. Gavin PJ, Suseno MT, Cook FV, Peterson LR, Thomson RB. Left-sided endocarditis caused by Pseudomonas aeruginosa: successful treatment with meropenem and tobramycin. Diagn Microbiol Infect Dis. 2003;47:427-30.

22. Paul M, Grozinsky S, Soares-Weiser K, Leibovici L. Beta lactam antibiotic monotherapy versus betalactamaminoglycoside antibiotic combination therapy for sepsis. Cochrane Database Syst Rev. 2006:CD003344.

23. Falagas $\mathbf{M}$, Matthaiou $\mathbf{D}$, Bliziotis $\mathbf{I}$. The role of aminoglycosides in combination with a beta-lactam for the treatment of bacterial endocarditis: a meta-analysis of comparative trials. J Antimicrob Chemother. 2006;57:639-47.

24. Safdar N, Handelsman J, Maki D. Does combination antimicrobial therapy reduce mortality in Gram-negative bacteraemia? A meta-analysis. Lancet Infect Dis. 2004; 4:519-27.
25. Kiffer CR, Mendes C, Kuti JL, Nicolau P. Pharmacodynamic comparisons of antimicrobials against nosocomial isolates of Escherichia coli, Klebsiella pneumoniae, Acinetobacter baumannii and Pseudomonas aeruginosa from the MYSTIC surveillance program: the OPTAMA program, South America 2002. Diagn Microbiol Infect Dis. 2004;49:109-16.

26. Nicolau DP. Pharmacokinetic and pharmacodynamic properties of meropenem. Clin Infect Dis. 2008;47:S32-40.

27. Keam SJ. Doripenem: a review of its use in the treatment of bacterial infections. Drugs. 2008;68:2021-57.

28. Horiuchi M, Kimura M, Tokumura M, Hasebe N, Arai $\mathrm{T}$, Abe K. Absence of convulsive liability of doripenem, a new carbapenem antibiotic, in comparison with beta lactam antibiotics. Toxicology. 2006;222:114-24.

29. Alexiou C, Langley SM, Stafford H, Lowes JA, Livessey SA, Monro JL. Surgery for active culture-positive endocarditis: determinants of early and late outcome. Ann Thorac Surg. 2000;69:1448-54.

30. Olaison L, Pettersson G. Current best practices and guidelines. Indications for surgical intervention in infective endocarditis. Infect Dis Clin N Am. 2002;16:453-75.

31. Thuny F, Di Salvo G, Belliard O, Avierinos JF, Pergola V, Rosenberg V, et al. Risk of embolism and death in infective endocarditis: prognostic value of echocardiography: a prospective multicenter study. Circulation. 2005;112:69-75.

32. Baddour LM, Wilson WR, Bayer AS. Infective endocarditis. Diagnosis, antimicrobial therapy, and management of complications: a statement for healthcare professionals from the Committee on Rheumatic Fever, Endocarditis, and Kawasaki Disease, Council on Cardiovascular Disease in the Young, and the Councils on Clinical Cardiology, Stroke, and Cardiovascular Surgery and Anesthesia, American Heart Association: endorsed by the Infectious Diseases Society of America. Circulation. 2005;111:e394-434.

33. Eishi K, Kawazoe K, Kuriyama Y, Kitoh Y, Kawashima Y, Omae T. Surgical management of infective endocarditis associated with cerebral complications. Multi-center retrospective study in Japan. J Thorac Cardiovasc Surg. 1995;110:1745-55.

34. Sexton D, Spelman D. Current best practices and guidelines. Assessment and management of complications in infective endocarditis. Infect Dis Clin N Am. 2002;16:507-21. 